\title{
Treatment of heterophoria with botulinum toxin A — a case study
}

\author{
Przemysław Raczkiewicz $\oplus^{1}$, Barbara Biziorek² \\ 'Student Research Group at the Clinic and Department of General and Children's Ophthalmology of the Medical University of Lublin, Lublin, Poland \\ ${ }^{2} \mathrm{OCHO}$ - Eye Surgery Center, Nałęczów, Poland
}

\begin{abstract}
BACKGROUND: Heterophoria (latent strabismus) is an imbalance in the tone of the oculomotor muscles. In a young child, this disorder usually does not affect the development of binocular vision. While looking, binocular vision (image fusion) keeps the eyeballs straight. With age, binocular vision may be impaired, for example, as a result of trauma, general disease, stress, or severe fatigue, and thus strabismus (heterotopia) may be revealed, which causes double vision. The patient, in our case, developed diplopia as a result of stress and fatigue.

CASE PRESENTATION: The patient underwent treatment with the use of botulinum toxin A. After each procedure, an ophthalmological examination was performed to measure the strabismus angle. Three courses of botulinum toxin A injection reduced the strabismus angle by $86 \%$, the double vision passed.

CONCLUSIONS: The case shows the possibility of curing an adult with Botulinum toxin A, in whom latent strabismus has turned into esotropia. Despite its transient kinetics, botulinum toxin A can have a lasting effect on eye alignment, favoring binocularity and reducing double vision, and can serve as a primary treatment. To obtain a permanent result, it is necessary to repeat the treatment.
\end{abstract}

KEY WORDS: strabismus; botulinum toxin A; esotropia

Ophthalmol J 2021; Vol. 6, 151-154

\section{INTRODUCTION}

Strabismus is a misalignment of one or both eyes, which may be intermittent or constant. It is a common condition that occurs in up to $5 \%$ of the population. In heterophoria, a relative deviation of the visual axes is held in check by fusion. In heterotropia, there is a manifest deviation of the visual axes. Strabismus can be further divided into esotropia (inturning deviation), exotropia (out-turning deviation), hypertropia (upturning deviation), hypotropia (down turning deviation), and cyclotropia (rotatory deviation). Strabismus can be caused by various insults such as abnormal anatomical development of extraocular muscles or the orbit, impaired neurological input to extraocular muscles, an uncorrected refractive error, or hereditary factors [1]. If present during a large part of childhood, it may result in amblyopia or loss of depth perception. If onset occurs during adulthood, it is more likely to result in double vision [2]. The extraocular muscles control the position of the eyes. Any issue with the muscles or the nerves that control them can cause paralytic strabismus [3]. 
Treatment depends on the type of strabismus and its cause. Non-surgical management of strabismus in adults includes optical treatment of refractive errors and prescription glasses, prisms, orthoptic exercises, and botulinum toxin A injections. Strabismus surgery attempts to realign the eyes by shortening, lengthening, or repositioning one or more extraocular muscles [4].

\section{CASE PRESENTATION}

The case shows the possibility of curing decompensated heterophoria with botulinum toxin $\mathrm{A}$ in an adult patient. The main aim of this study is to show the effectiveness of the botulinum toxin A therapy in treating convergent strabismus with low fusional reserves.

A 20-year-old man consulted a doctor with paroxysmal headaches, fatigue, problems with concentration and reading. As a first-year student, the patient had recently increased mental effort, and these pain symptoms disturbed his functioning. Internal and neurological examinations did not reveal any pathologies. Blood tests showed that iron, glucose, thyroid-stimulating hormone (TSH), vitamin D3, creatinine, morphology, and urea levels were within the standard limits. The patient was referred for an MRI of the head with contrast. The radiographic examination was normal, and the structures of the brain remained unchanged.

The patient went to the optometrist. Ophthalmological examination showed latent strabismus and low fusional reserves. Worth's test indicated near fusion, while to the distance, there was uncrossed diplopia. A prismatic correction was prescribed to alleviate discomfort and to compensate for latent strabismus-heterophoria (Tab. 1). The patient also signed up for visual training.

After a month, an eye examination was performed. The strabismus angle increased to $14,5 \Delta$ esotropia. In addition, the patient reported having diplopia after removing the glasses. There was a decompensation of latent strabismus, and heterotropia appeared. The patient was unable to adapt to the prismatic correction. Headaches and problems with concentration while working at close range increased. The patient was referred to the Eye Surgery Center of prof. Zagórski in Lublin for further diagnosis and treatment.

In the ophthalmological examination, he was diagnosed with convergent concomitant strabismus. The anterior and posterior segments of the eye were without pathological changes. The visual acuity of the left and right eye was 1.0. When the patient was tired, the left eye escaped inward (Fig. 1). The decision was made to treat him with botulinum toxin $\mathrm{A}$.

On 11 October 2018, a procedure was performed: injections of botulinum toxin A (Botox ${ }^{\circ}$, Allergan Pharmaceuticals) under the control of an acoustic electromyograph into the right medial rectus muscle 0.6 units and the left medial rectus muscle 1.25 units. After the procedure, the patient stopped using the prismatic correction. The spherical correction remained unchanged (Fig. 1). One month later, during the post-operative examination, the strabismus angle was $7 \Delta$ exophoria. Transient overcorrection and the transition from convergent to divergent strabismus is the desired result. A normally functioning antagonist muscle prevails over the paralyzed, treated muscle. This stimulates muscle remodeling to maintain the correct alignment of the eye muscles (orthophoria) [9].

On 21 February 2019, an ophthalmological examination was performed. The strabismus angle was $6 \Delta$ esophoria. The eyes converged back from the divergent position. The patient reported a significant improvement after the procedure: headaches, difficulties in concentrating, and the double vision passed.

On 7 March 2019, another botulinum toxin A injection procedure was performed. Under the control of the acoustic electromyograph, 1.25 units

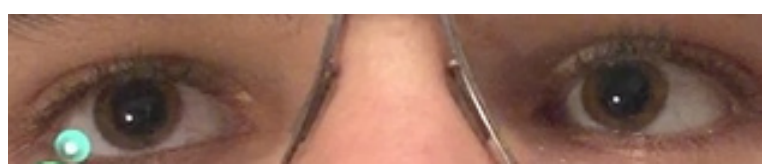

FIGURE 1. Decompensation of latent strabismus due to fatigue

\begin{tabular}{|l|c|c|c|c|c|}
\hline Table 1. Prescription for glasses \\
\hline & Sphere & Cylinder & Axis & Prism & Base \\
\hline Right eye & -1.25 & $-0,5$ & 180 & $5 \Delta$ & 180 \\
\hline Left eye & -1.25 & -0.5 & 170 & $5 \Delta$ & 0 \\
\hline
\end{tabular}




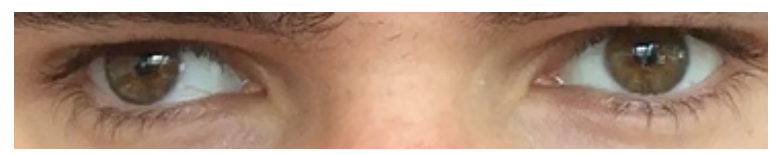

FIGURE 2. Exotropia after botulinum toxin A injection

of botulinum toxin A were injected into the medial rectus muscle of the right eye and 1.25 units to the medial rectus muscle of the left eye. Examination performed on $03 / 28 / 2019$ showed distance vision $32 \Delta$ exotropia and near vision $12 \Delta$ exotropia. The patient preserved stereoscopic vision despite the divergent eye position (Fig. 2).

On 28 May 2019, an ophthalmological examination was performed. The strabismus angle was $12 \Delta$ exophoria to distance vision and $4 \Delta$ exophoria to near vision. The patient stopped reporting side effects related to the botulinum toxin A. He claimed that never before had he experienced such good working comfort when working at close range.

On 20 February 2020, another ophthalmological examination was performed. The strabismus an-

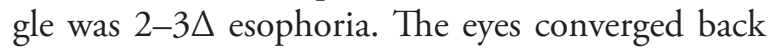
from the divergent position.

On 27 February 2020, yet another botulinum toxin A injection treatment was performed. Under the control of the acoustic electromyograph, 0.6 units of botox were injected into the medial rectus muscle of the left eye.

On 7 July 2020, a subsequent ophthalmological examination was performed. The strabismus angle was $2 \Delta$ esophoria. The fusional reserves were $6 \Delta$. The obtained effect is constant; the strabismus angle was decreased by $86 \%$. Binocular vision was preserved in the examinations. Worth's test indicated distance and near fusion in both bright and dark lighting (Fig. 3).

\section{DISCUSSION}

In 1989 the United States Food and Drug Administration (FDA) approved botulinum toxin A therapy to treat strabismus in people over 12 years of age [5]. This provided an alternative to surgery. This technique, most commonly used in adults, is also used to treat children, especially children affected by childhood esotropia. Research using botulinum toxin have proved this drug is effective in the the following diseases: esotropia and exotropia with angle deviation smaller than $40 \mathrm{PD}$, sixth nerve palsies (mitigating the effects of acute paralytic strabismus), postoperative strabismus, ac-

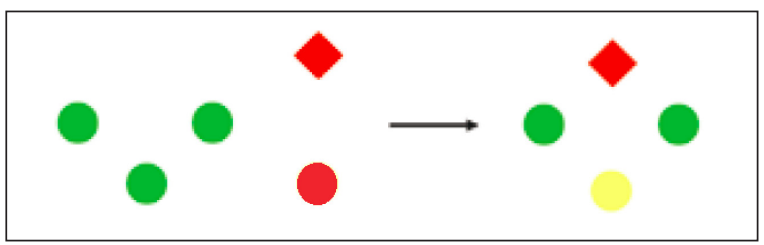

FIGURE 3. Worth's Four Dot test after three botulinum toxin A treatments

tive thyroid eye disease [10]. Favorable treatment results are obtained in the following case: small to moderate deviations, young age patients, potential for binocular vision, bilateral eyes muscles injections [11]. Treatment of small deviations gives the best result, as also seen in the present study. Deviation above $40 \Delta$ should not be treated with botulinum injections [14].

Botulinum toxin $\mathrm{A}$ is a drug produced by the bacterium Clostridium botulinum. This action mechanism is based on blocking the release of acetylcholine from cholinergic synapses found in the muscle, thereby blocking nerve impulses and preventing muscle cell contraction. When a muscle is paralyzed, the opposite muscles take on more force to move, and the eye position straightens. The toxin is injected into the stronger muscle, causing temporary and partial paralysis. Treatment may need to be repeated three to four months later when the paralysis is resolved. Common side effects include double vision, drooping eyelid, overcorrection, and no result. However, side effects usually disappear within three to four months [6].

The first significant study of botulinum toxin in strabismus was published by Scott et al. in 1990 [13]. This study examined the results of botulinum toxin treatment of 362 children and showed that $61 \%$ of children maintained an alignment correction within $10 \Delta$ [12]. In one study, the ocular deviation was reduced by an average of $60 \%$ in an adult group [15]. To compare, in our patient, strabismus was reduced by $12.5 \Delta(86 \%)$ to $2 \Delta$ esophoria. In 2010, Campomanes et al. conducted a prospective comparative study. They found that in patients with a deviation of less than $30 \Delta$, the success rate of botulinum toxin $A$ treatment was the same as for incision surgery [7]. Studies show a reduction in the strabismus angle by $70 \%$ in children with esotropia and 54\% in monocular exotropia after treatment with botulinum toxin A [8].

In this case, the botulinum toxin $\mathrm{A}$ injection was used due to the lack of conservative treatment results such as prisms glasses and orthoptic exercis- 
es. The case shows that botox can be used in latent strabismus (heterophoria), which turns into esotropia during fatigue. After injection of botulinum toxin A, esotropia turns into exotropia. A temporary overcorrection is desirable in treatment with botulinum toxin $\mathrm{A}$.

\section{CONCLUSIONS}

The strabismus angle was too small for a surgical operation. The use of botulinum toxin A turned out to be more advantageous than conservative treatment (optometric exercises, prism glasses). Botulinum toxin A can permanently affect the eye's position (in this case, it reduced the strabismus angle by $86 \%$ ), promoting binocularity and reducing double vision. It can be used as a basic treatment, although it is necessary to repeat it to obtain the permanent effect.

\section{Acknowledgments}

The completion of this study could not have been possible without several ophthalmological examinations conducted by Monika Hereć, PhD. Many thanks for your engagement.

\section{Conflicting interests}

The authors declare that there is no conflict of interest.

\section{REFERENCES}

1. RoweFJ, Noonan CP. Botulinum toxin for the treatment of strabismus. Cochrane Database Syst Rev. 2017; 3: CD006499, doi: 10.1002/14651858. CD006499.pub4, indexed in Pubmed: 28253424.
2. Gunton $K B$, Wasserman $B N$, DeBenedictis C. Strabismus. Prim Care. 2015; 42(3): 393-407, doi: 10.1016/.jpop.2015.05.006, indexed in Pubmed: 26319345.

3. Cunningham ET, Riordan-Eva P. Vaughan \& Asbury's General Ophthalmology. 18th ed. McGraw-Hill Medical, Huckelberry 2011.

4. MacEwen C, Gregson R. Manual of strabismus surgery. ButterworthHeinemann, Oxford 2003.

5. Docket No. FDA-2008-P-0061. Food and Drug Administration. United States Department of Health and Human Services.

6. Thouvenin D, Lesage-Beaudon C, Arné JL. Injection de toxine botulique dans les strabismes précoces. Efficacité et incidence sur les indications chirurgicales ultérieures. J Franç Ophtalmol. 2008; 31(1): 42-50, doi: 10.1016/s0181-5512(08)70329-2.

7. de Alba Campomanes AG, Binenbaum G, Campomanes Eguiarte G. Comparison of botulinum toxin with surgery as primary treatment for infantile esotropia. J AAPOS. 2010; 14(2): 111-116, doi: 10.1016/j. jaapos.2009.12.162, indexed in Pubmed: 20451851.

8. Mangan MS, Basar E, Mahan M, et al. Pediatric Eye Disease Investigator Group. Botulinum A toxin therapy on esotropia in children. J Med Assoc Thai. 2002; 85(11): 1189-1197, indexed in Pubmed: 12546316.

9. Scott $A B$. Change of eye muscle sarcomeres according to eye position. J Pediatr Ophthalmol Strabismus. 1994; 31(2): 85-88, indexed in Pubmed: 8014792.

10. Hered R. Chapter 14: Surgery for extraocular muscles. In: In: 20182019 Basic and Clinical Science Course, Section 06: Pediatric Ophthalmology and Strabismus. American Academy Ophthalmology, Philadelphia 2018: 241-242.

11. Dressler $D$. Clinical presentation and management of antibody-induced failure of botulinum toxin therapy. Mov Disord. 2004; 19 Suppl 8: S92S9S100, doi: 10.1002/mds.20022, indexed in Pubmed: 15027060.

12. Rayner SA, Hollick EJ, Lee JP. Botulinum toxin in childhood strabismus. Strabismus. 1999; 7(2): 103-111, doi: 10.1076/stra.7.2.103.650, indexed in Pubmed: 10420215.

13. Scott A, Magoon E, McNeer K, et al. Botulinum Treatment of Childhood Strabismus. Ophthalmology. 1990; 97(11): 1434-1438, doi: 10.1016/ s0161-6420(90)32390-4, indexed in Pubmed: 2255516.

14. Lennerstrand G, Nordbø OA, Tian $S$, et al. Treatment of strabismus and nystagmus with botulinum toxin type $A$. An evaluation of effects and complications. Acta Ophthalmol Scand. 1998; 76(1): 27-27, doi: 10.1034/j.1600-0420.1998.760106.x, indexed in Pubmed: 9541431.

15. Elston JS, Lee JP, Powell CM, et al. Treatment of strabismus in adults with botulinum toxin A. Br J Ophthalmol. 1985; 69(10): 718-724, doi: 10.1136/bjo.69.10.718, indexed in Pubmed: 4052354. 